\title{
A Visual Analytics System for Route Planning and Emergency Crowd Evacuation
}

\author{
Saleh Basalamah \\ Umm Al-Qura University \\ Makkah, Saudi Arabia
}

\begin{abstract}
Emergency evacuation from crowded public spaces is of great importance to all authorities around the world. Many systems have been developed by researchers to address the optimization of emergency evacuation routing and planning. This paper presents a visual analytics system for route planning and emergency crowd evacuation; a web-based visualization and simulation system that allows stakeholders to develop and assess route planning and evacuation procedures for emergency scenarios. The system takes advantage of the available OpenStreetMap comprehensive spatial database to enable users to implement evacuation scenarios in almost anywhere OpenStreetMap dataset is available. Using multiple infrastructure-specific varying scenarios, such as adjusting capacities of roads/pathways or their closure, the tool can identify bottleneck areas thus allowing the assessment of potential improvements to pedestrian and transportation network to relieve the bottleneck and improve evacuation time. As a case study, we use this system for the city of Makkah in Saudi Arabia and the city of Minnesota in the United States.
\end{abstract}

Keywords-Emergency evacuation; crowd management; visualization; intelligent systems

\section{INTRODUCTION}

Emergency evacuation is the movement of a large number of people from one place to another in a short period of time in response to an emergency. This is due to different types of emergencies; natural disasters, terrorism, stampede or war. Throughout the years, many emergency evacuation incidents occurred which needed mass evacuation. Planning of evacuation scenarios plays an important factor in the success of a mass emergency evacuation and many evacuations failed for the lack of good planning. Authorities in different parts of the world provide guidelines and help materials for people to use in case of an emergency evacuation; e.g. www.ready.gov

Integrated decision support systems are used to assist authorities to respond to emergencies in an optimized way [1]. They support strategic and operational decision making in terms of using resources and planning scenarios.

In this regards, we present a visual analytics system for evacuation route planning. Most of the small and large-scale events require an emergency evacuation tool to handle any disaster conditions including flood, fire, or any terrorist attack. The visual analytics system helps to identify the evacuation routes and time based on number of evacuees. This system helps authorities to make a plan to handle any emergency scenarios by changing number of evacuees, shelter points and by closing or expanding some paths. This will help decisionmakers in the selection of optimized number of shelters, maximum number of evacuees that can be evacuated in a given time, impact on time and flow of the evacuation plan by closing or expanding some roads. Unlike existing systems, our system is generic and easily applicable to any outdoor events. The system takes advantage of the available OpenStreetMap (OSM) comprehensive spatial database to enable users to implement evacuation scenarios in almost anywhere OSM dataset is available. The system converts OSM dataset into spatial relational database. Moreover, our system allows end user to enhance the spatial relational database interactively such as assigning the width of each road to calculate the capacity, source point, number of evacuees in each source, shelter points and capacity of each shelter point. The visual output of the evacuation routes and time graph makes the system more convenient for analytics. To prove our system capabilities, we demonstrate the system for the city of Makkah, Saudi Arabia and Minnesota, United States of America.

\section{LITERATURE REVIEW}

Emergency situations like fires, floods, earthquakes and terrorist attacks pose threat to the people living at the vicinity. A system is therefore needed to help the stakeholders and decision-makers to be in the state of readiness to efficiently and effectively evacuate large crowd from such areas of threat. The state-of-the-art [2] of routing algorithms for static and continuous-time network produces paths and plans, however, these approaches are confined to building and small areas. Similarly, the routing algorithms for single-source shortest path, all-pair shortest path, time dependent network provide nearest and shortest destination and time but neglects road capacity constraints [3]-[5]. Likewise, work done in [6]-[8] are restricted to evacuating buildings. Moreover, evacuation of evacuees in building has been done using wireless sensor networks with the demonstration of simulation on static data [9]. Evacuating crowds on a large scale was studied using the Capacity-Constrained Route Planning (CCRP) [10], [11], nearest exist or shelters (NES) [12], and crowd-separated allotment of routes and shelters (CARES) [13].

CCRP heuristic approach algorithms [10], [11] contemplates on shortest path algorithms with capacity constraints of each node and edge of the road. Nearest terminus along with the edge capacity is considered by NES [12] algorithm but it violates the destination capacity. CCRP and NES can result in movement turbulences and stampede in case of evacuating huge crowd. The algorithm, CARES [13], overcomes the drawbacks of above mentioned algorithms and takes into consideration the capacity constraint along with spatial anomaly to avoid movement turbulence and stampede. Haj, results in huge gathering every year in the city of Makkah, and the 
CARES algorithm demonstrated the evacuation of large crowd in pedestrian mode very well.

Our proposed visual analytics system for route planning and evacuation uses CARES [13] algorithm. The system, can work on any part of the world where OSM data set is available. Instead of developing from the scratch for different venue, the proposed system allows end-user to do little tuning by providing details about the roads along with the number of evacuees, source and destination points.

\section{System OVERVIEW}

The architecture of the proposed system is depicted in Figure 1, which consists of two systems; a backend-system and a front-end web-based visualization system. Towards the development of these two systems, two tasks have been pursued.

\section{A. Back-end Database and Computational System}

We have developed and tested an integrated architecture of the back-end system of the proposed tool that consists of various databases maintaining data from numerous channels, depicted in Figure 1. Using the OpenStreet map, the tool generates a graph modeling the road network for the area under consideration for evacuation. The travel time of each segment of a road/street is computed based on the end points of the segment. Roads are classified and tagged according to their width. Subsequently, the capacity of each road is stored in a table based on its type. Such type-capacity table is provided by the user. A key component of the backend system is the CARES algorithm which accesses the backend databases and generates the evacuation plan for the selected area by identifying routes to a set of pre-selected shelters. Evacuation scenarios are orchestrated through the front-end visual interface which allows a user to select various sites from the digital map database requiring evacuation. In addition, it allows the user to select the destination sites for the evacuees. Such interaction between the user and the proposed system is supported by the front-end system. The user can interactively specify constraints specific to route planning during emergency evacuation, prior to the execution of the CARES algorithm. Constraints can include partial/full closure of some roads/streets from the map which may have blockage, due to construction or impact of on-going emergency, such as growing level of flooding. The pre-computed values for capacities of routes and the travel times based on the physical environment due to the changing context, are utilized by the system. The physical environment depicts the geo-spatial characterization of a road/pathway in terms of the degree of its curvature, its uphill/downhill slope, the width, and blockages etc. For this purpose, the system uses the route map of the city under consideration, the City of Makkah and the Hajj premises, in our case. A high-level pseudo-code for CARES algorithm is given in Algorithm. 1.

\section{B. Front-end System}

In this task have developed the front-end visual analytics system to analyze the various emergency scenarios and preplanning exercise for emergency evacuation, as well as decision support capability for route planning, as a part of the pre-planning exercise. The system provides multi-level linked

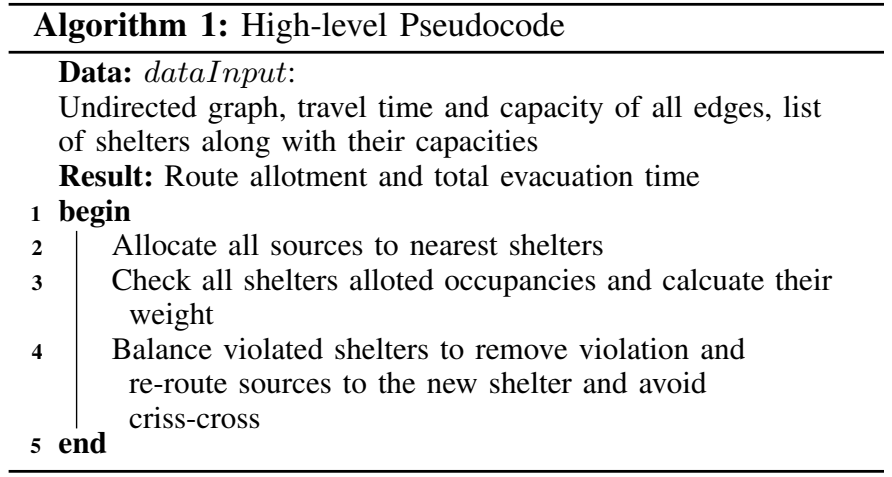

views and interactive displays that allow interactive analysis, as shown in Figure 2. User can provide input to generate various evacuation scenarios. These scenarios comprise of the point(s) of incident and number of evacuees at each point of incident. Potential destination sites for the evacuees can be identified in the city of Makkah based on Metro land road connectivity including Jamaraat, Arafat, and Kudai Parking. Based on crowd modeling given in [15], [16], we have identified the capacity and travel speed as shown in the table in Figure 3.

The tool allows users to assess potential increase or decrease in risks associated with selecting evacuation sites in terms of performance factors including total evacuation time, potential number of people evacuated, and management of routes. In essence, the tool can provide a thorough assessment of all evacuation operations conducted by the Ministry of Hajj. In addition, the tool's functionality can be extended to provide officials an automated capability to make decisions about optimal allocation of evacuation sites under various emergency scenarios.

\section{SCALABILITY}

The scalability is one of the important aspect of the system. It becomes more challenging when the system is related to evacuation. In evacuation cases, there are too many variations starting from small area or stadium to an entire city. To make the system scalable, the evacuation system should be intelligent enough to handle all such scenarios. The proposed system assuming $\mathrm{n}$ is the number of nodes (road intersections), $\mathrm{m}$ is the number of edges (roads), $s$ is the number of shelters, and $p$ is the number of evacuees, the computational complexity of the tool is

$$
O(p * s *(n * \log (n))) .
$$

Since the complexity is sub-quadratic in network size and the number of evacuees, the system provides a scalable strategy for evacuation planning. The extraction of nodes and edges of the data from OSM are discussed in next section.

\section{Graph Generation and Scenario Selection}

In this section, we demonstrate several functionalities of the tool. These functionalities illustrate both the intelligent component (the back-end system) and the visual analytics capability. Following are the database functions that have been implemented in the backend system and are required to run the tool. 


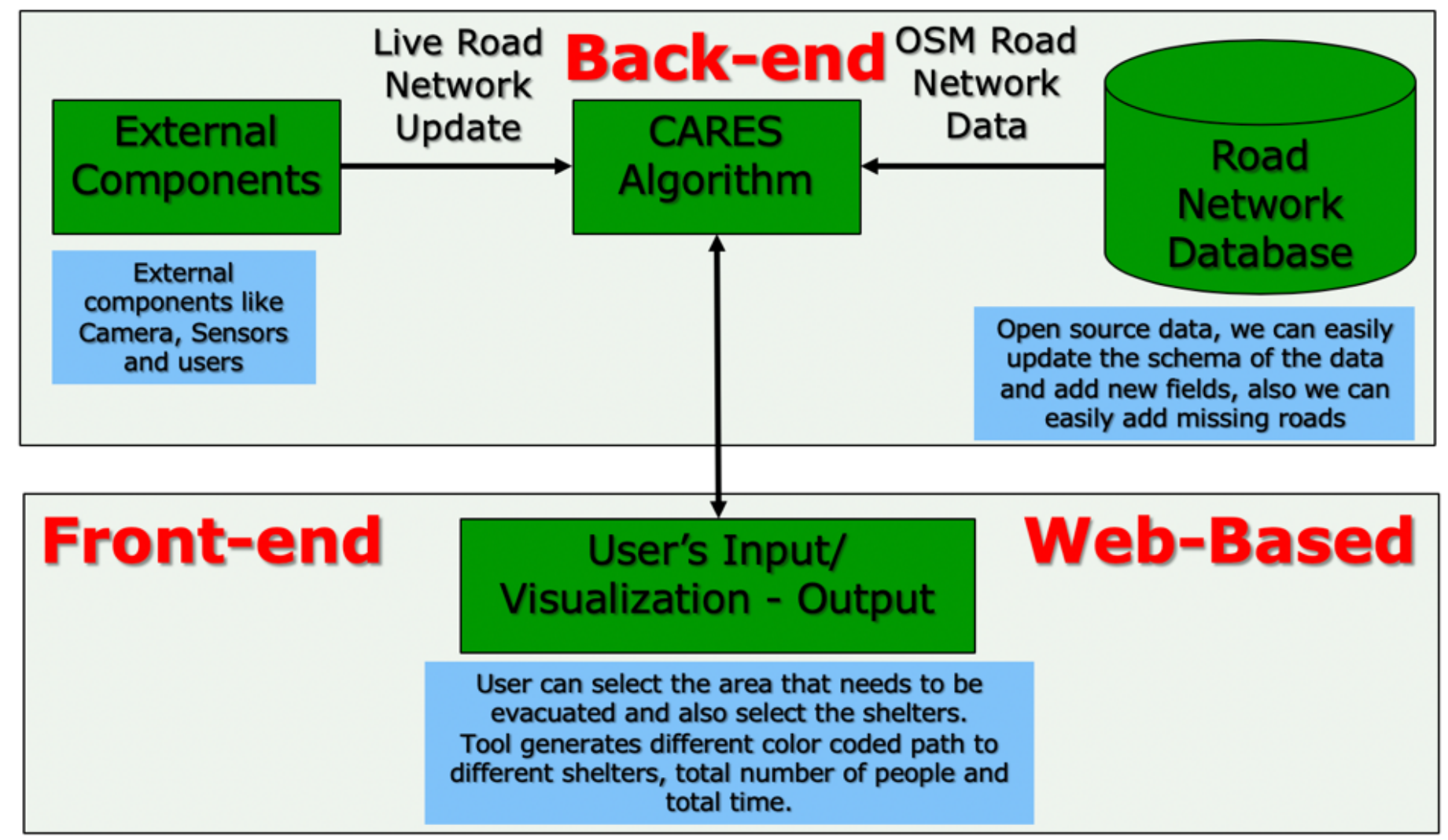

Fig. 1. Overall architecture of the proposed system

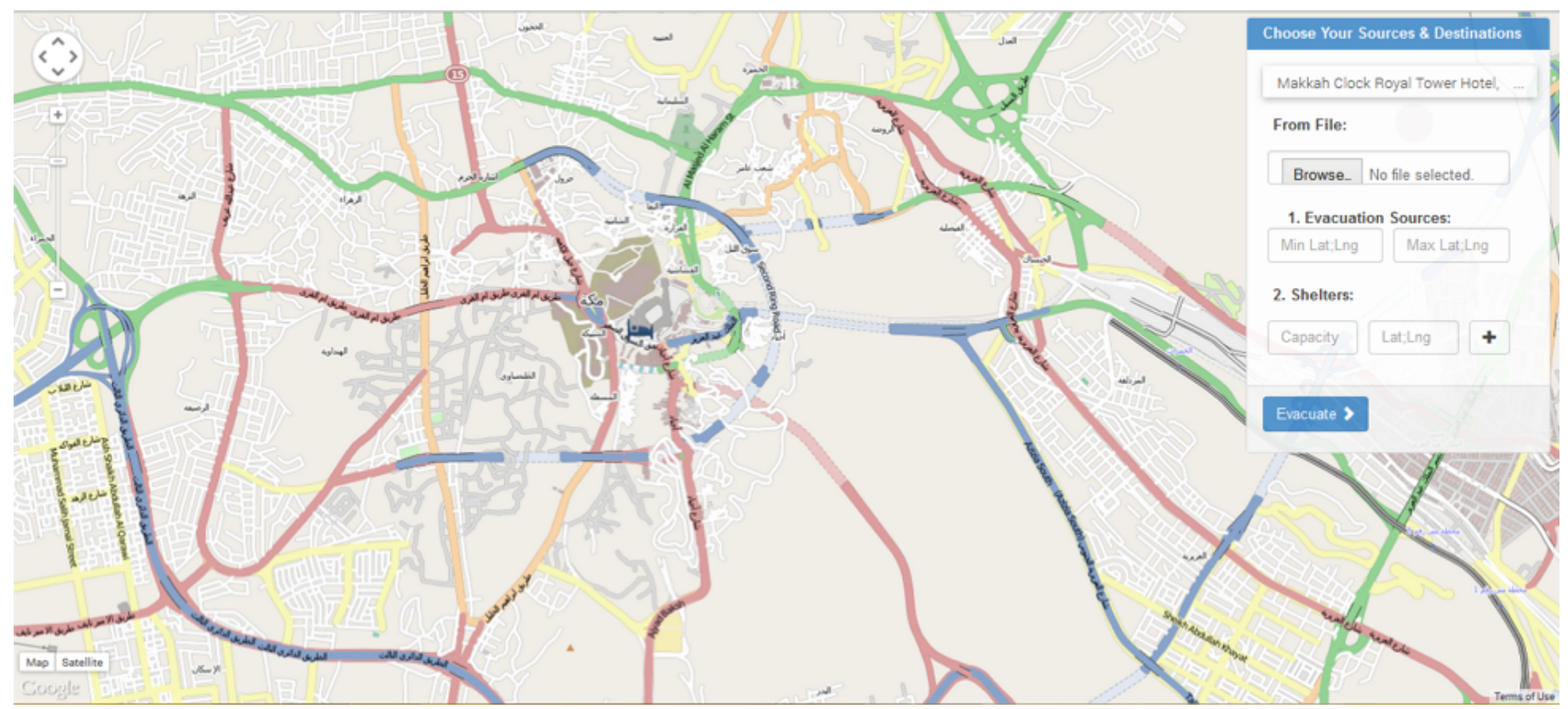

Fig. 2. Visual analytics interface of the system. 


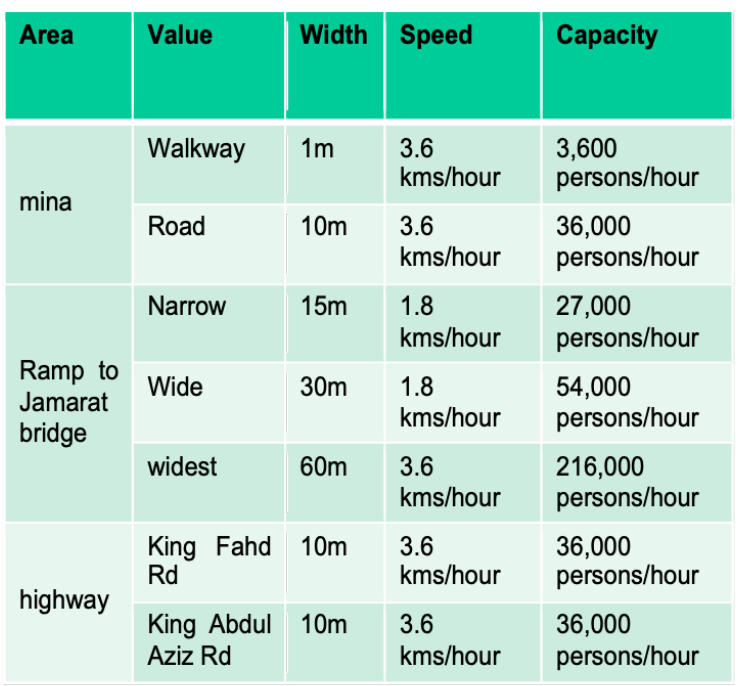

Fig. 3. Speed and Capacity.

\section{A. Database Generation}

1) Fetching Data from OSM: The system fetches the data of any city under consideration (city of Makkah, Saudi Arabia for the case study) from Open Street Map (OSM). The OSM provides the data in a PBF/XML format that requires preprocessing to convert the data into required format along with the cleaning. TAREEG [14] uses OpenStreetMap data set and develops a web-service that extracts spatial data from any part of the world easily using MapReduce-based techniques. TAREEG is scalable and removes the irrelevant noisy data and allows the user to download the data in multiple format. In the proposed system, the same approach of TAREEG has been used to extract the data of OSM dataset in edges and nodes relational format by selecting the area on the map as shown in Figure 4.

2) Converging of Data into Road Network: OSM provides the data in XML/PBF format. By using OSM2pgSQL tool, the backend system converts the OSM into a graph model, $\mathrm{G}=$ $(\mathrm{V}, \mathrm{E})$, where $\mathrm{V}$ represent the set of vertices and $\mathrm{E}$ is the set of edges. The tool is helpful to extract road relation database of the selected area.

3) Assignment of Average Travel Time and Capacity: Subsequently tool runs a time and capacity module that provides the time and capacity values for each edge of the graph model. Table I shows different types of roads that are retrieved from Open Street Map. We assume an average speed (for all types of pedestrians on all types of roads) of $1 \mathrm{~m} / \mathrm{s}$ [13]. The default capacity is assigned based on the type of the road. The system also allows user to change capacity of the road by editing the width of the road. The width of the road differ from city to city and its an important parameter that is taken into consideration while making the evacuation plan.

\section{B. Evacuation Scenario Generation}

We have successfully extracted and loaded the complete road network with all the nodes and edges from Open Street map. As mentioned earlier, Open Street map is an open source
TABLE I. DIFFERENT TYPES OF ROADS

\begin{tabular}{|c|}
\hline Types of Road \\
\hline Motorway \\
\hline Trunk \\
\hline Tertiary \\
\hline Primary \\
\hline Secondary \\
\hline Residential \\
\hline Unclassified \\
\hline
\end{tabular}

and can be easily modified by adding new column in the table such as capacity and travel time. In some cases, we need to add more nodes and create new edges to provide further detail about the routes, for example, for the area of Mina. Figure 5 shows various nodes and edges for Mina. The corresponding graph model, in terms of nodes and edges are displayed through the front-end visual interface as depicted in Table II and Table III. Capacity and travel times are added to nodes and edges, respectively based on the approach provided in [15], [16].

As mentioned earlier, the tool provides a robust visual interface that allows the user to orchestrate an evacuation scenario encompassing several options depicting scale and the location of evacuation area, along with various other parameters. Through the following demo illustrations, we provide the detail of this important visual capability of the tool.

\section{Selection of Evacuation Area}

The user can identify the area to be evacuated in form of grid by visual demarking the area as depicted in Figure 6.

\section{Interactively Changing Road Conditions}

The tool also provides an option to the user (or emergency management authorities acting as users) to change the road plan by closing or expanding a road (or set of arbitrarily selected roads) on the map, as an additional capability for orchestrating a scenario. 


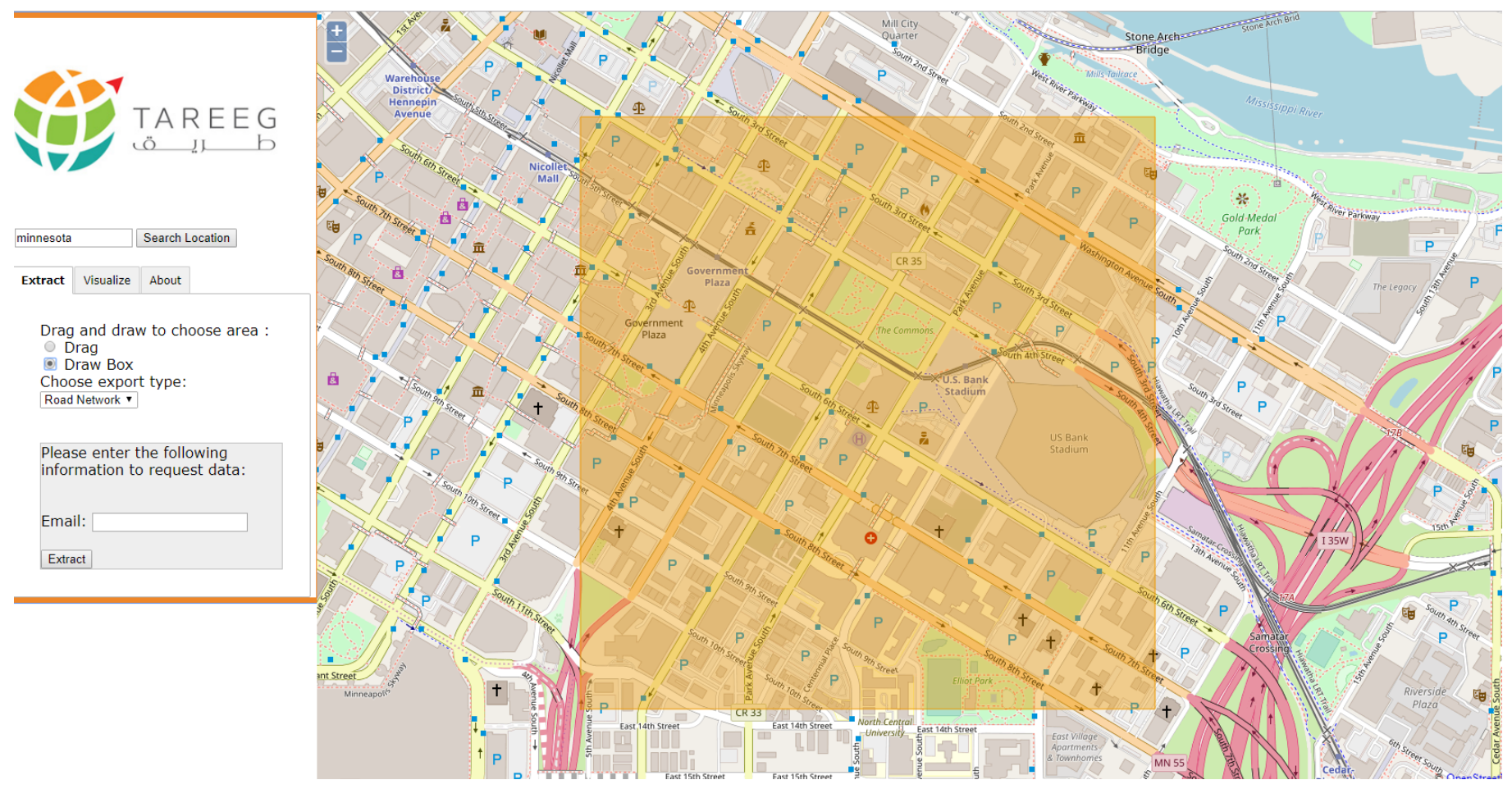

Fig. 4. Generic Tool to extract OpenStreetMap [14].

TABLE II. EXAMPLE OF THE DATA FOR NODE TABLE

\begin{tabular}{||c|c|c|c|c|c||}
\hline id & name & lat & lon & isShelter & capacity \\
\hline \hline 1 & 1114915162 & 21.414340 & 39.862888 & FALSE & 0 \\
\hline 2 & 1123952383 & 21.414178 & 39.858879 & FALSE & 0 \\
\hline 3 & 1123952396 & 21.417453 & 39.859701 & FALSE & 0 \\
\hline 4 & 1123952398 & 21.415552 & 39.859227 & TRUE & 2000 \\
\hline 5 & 1123952400 & 21.415031 & 39.858813 & FALSE & 0 \\
\hline
\end{tabular}

TABLE III. EXAMPLE OF THE DATA FOR EdGE TABLE

\begin{tabular}{||c|c|c|c|c|c|c||}
\hline id & name & sid & did & roadtype & time & capacity \\
\hline \hline 19627 & 304911020 & 1471778539 & 1471741582 & "highway"="residential" & 72 & 144 \\
\hline 19630 & 380891295 & 1471741582 & 1471741580 & "highway"="residential" & 195 & 390 \\
\hline 19631 & 380908949 & 1471741581 & 1471741579 & "highway"="residential" & 74 & 148 \\
\hline 19744 & 380891308 & 1471741600 & 1471733686 & "highway"="residential" & 185 & 370 \\
\hline 19750 & 380874956 & 1471741587 & 1471733682 & "highway"="residential" & 18 & 36 \\
\hline
\end{tabular}

\section{Visualizing And Assessing Route Planning AND EVACUATION PERFORMANCE}

The back-end algorithm in the tool runs in such a way that it avoids criss-crossings, which in turn helps the crowd to avoid another disaster or congestion. For example: if the evacuation output is from source ' $\mathrm{s}$ ' to destination ' $\mathrm{d}$ ' passing through multiple nodes $\mathrm{N}=\mathrm{n} 1, \mathrm{n} 2, \mathrm{n} 3 \ldots \mathrm{n}$ then all the evacuees on intermediate nodes ' $\mathrm{N}$ ' will move for evacuation towards destination ' $d$ ' only [13]. The results presented in this section illustrate how the performance of the tool, measured in terms of rate of evacuation, i.e. the number of evacuates arriving at the designated sheet(s) as a function of time. In particular, the effect of the following user's selected scenarios on the performance is presented. These visual outputs can be simultaneously and synchronously shared among distributed end-users.
This system gives the ability to decision-makers to assess different scenarios of evacuation by visualizing the change in routes and evacuation time. The decision-makers leverages the ability of the interactive system to visualise the effect of distinct scenarios such as effect of blocking or expanding the roads, effect of spreading and clustering the given number of shelters, effect of increasing the number of shelters and evacuees.

\section{A. Effect of Blocking Roads or Expanding the Capacity of Roads}

To identify the impact of closing or expanding the road is very critical. The tool is helpful to identify the impact of the road while evacuating the people with respect to time. Results displayed in Figure 7 show the effect on the overall evacuation time after closing the road. In particular, graph on the right 


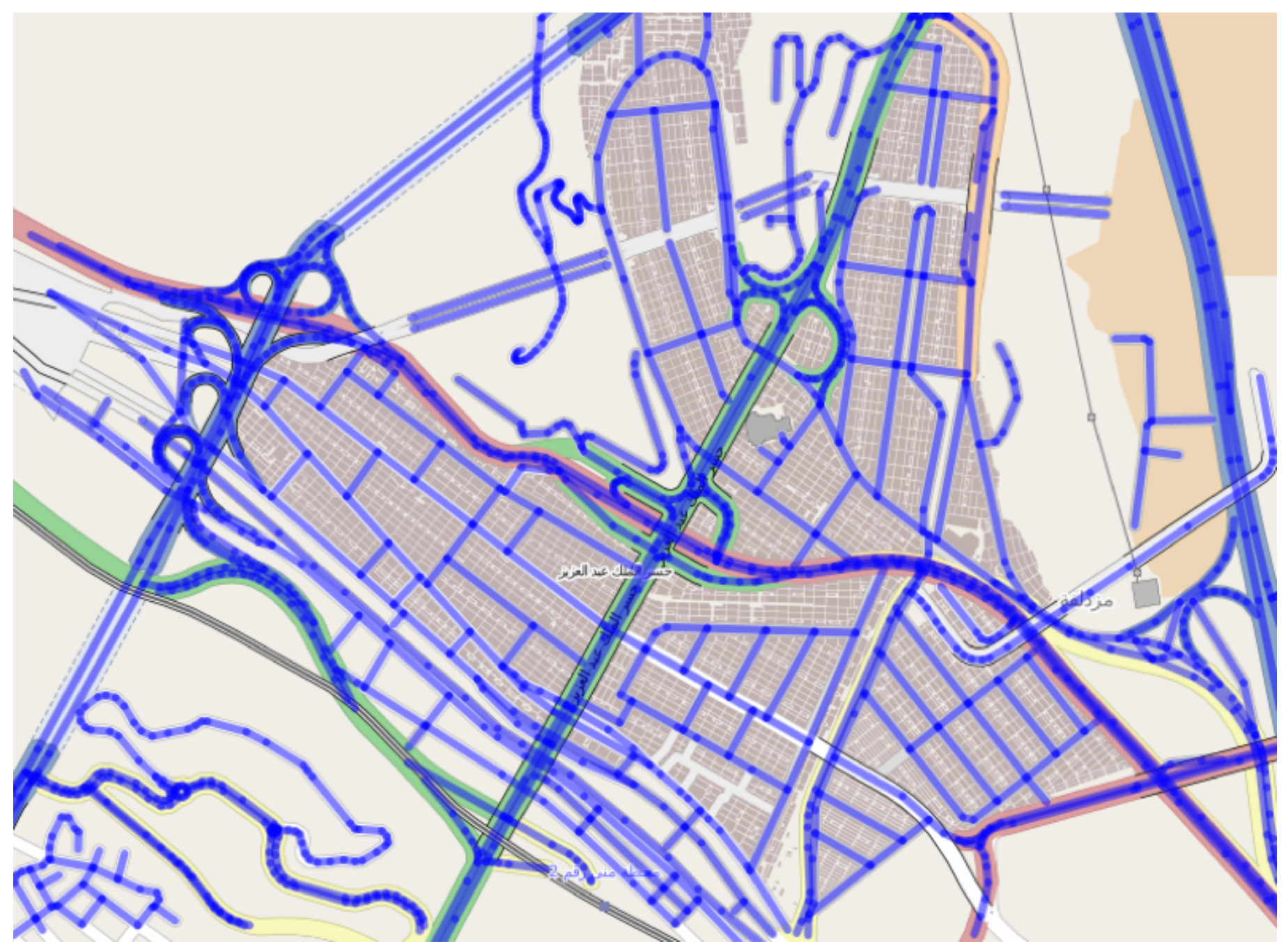

Fig. 5. Detailed Route Map for Mina.

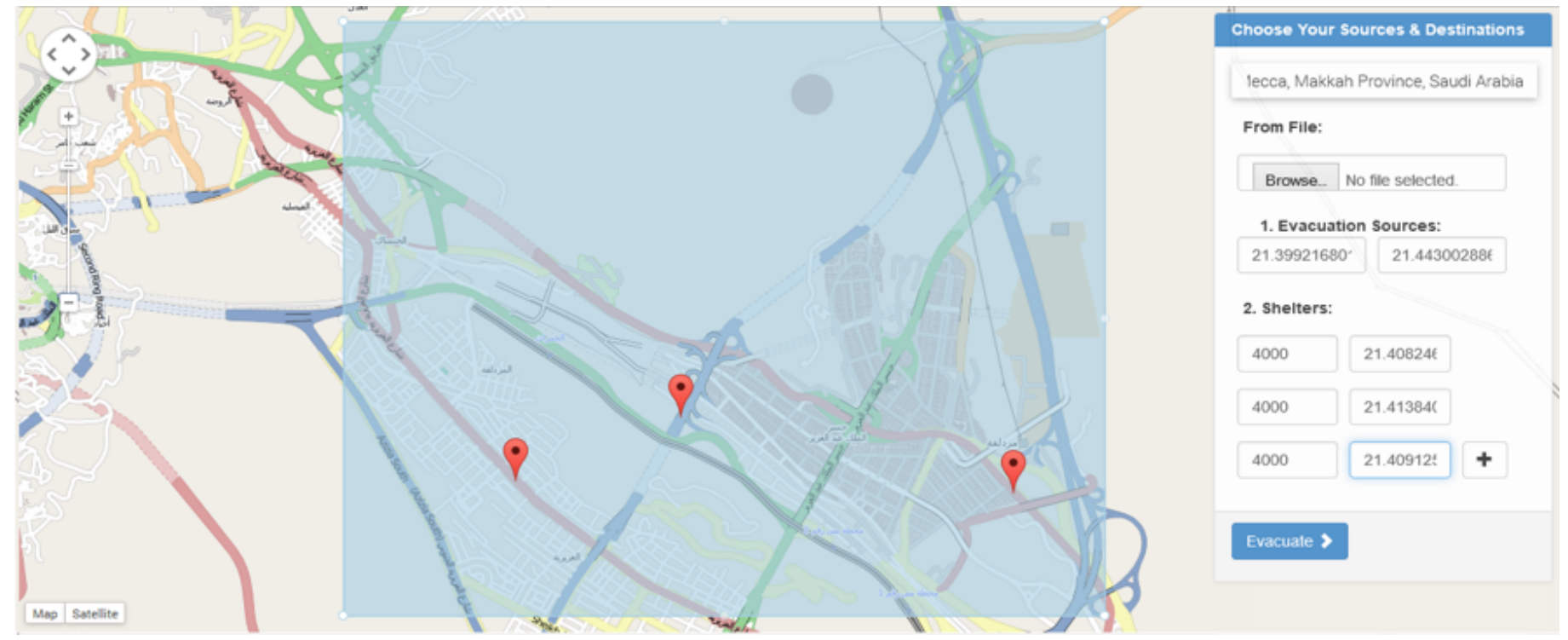

Fig. 6. Grid selection to demark the evacuation area with shelter points shown in red markers. Capacity is assigned to each shelter. 
shows the impact on the evacuation time after closing the road. The increase in the evacuation is noticeable, as expected under this scenario. By closing the major highway the evacuation time almost doubled from 4435 to 9149 seconds. The system allows decision-makers to take the decision by blocking or expanding the roads and visualize the effect on the evacuation time and routes.

\section{B. Effect of Increasing the Number of Evacuees}

The tool can be used by the end-user for evacuating different size of population. The output of the tool (exemplified in Figure 8) can provide an assessment whether or not the given population can be evacuated within a certain time frame, with the given size and capacity of the road network and shelters. Through such analysis, decision can be made about the limit on the size of the population that can be accommodated in the region on interest.

\section{Effect of Spreading/Clustering a Given Number of Shelters}

By running multiple scenarios, the tool can depict the difference in the evacuation time as the shelters are clustered or spread. Figure 9 shows the output with clustered shelters. Figure 9 and Figure 10 show the timing output for the case when the shelters are clustered close together vs. spreading them out. This allows decision-makers to identify the optimum location of shelter points to evacuate desired number of evacuees in a quick and an efficient manner.

\section{Effect of Increasing the Number of Shelters}

Figure 11 shows the impact of increasing the number of shelters on the evacuation time. Graph on the right shows the output with 5 shelters and graph on the left with 3 shelters shows the evacuation time 722 seconds and 943 seconds respectively to evacuate 23810 evacuees. Such type of assessment can enable the end-user to take the decision on the number of shelters to be allotted for a given scenario.

\section{CONCLUSION}

In this paper we propose a system for route planning and emergency crowd evacuation. We describe in detail the various capabilities of the tool, such as: scalability of route generation, visual assessment of various scenarios, along with demo outputs of Makkah, Saudi Arabia and Minnesota, USA. The proposed system utilizes Spatial database of the OpenStreetMaps and allow users to execute evacuation wherever dataset of the OpenStreetMaps are available. The capabilities of this tool can be expanded in various dimensions. For example, for realtime applications, it can be integrated with multi-modality data streams, such as live camera feeds, mobile cell data etc. In addition, the tool can be integrated with existing crowd and traffic management tools currently available.

\section{ACKNOWLEDGMENT}

This research was funded by the Center of Research Excellence in Transportation and Crowd Management, Umm Al-Qura University, Saudi Arabia.

\section{REFERENCES}

[1] Christian Artigues, Emmanuel Hébrard, Yannick Pencolé, Andreas Schutt, Peter Stuckey. A Study of Evacuation Planning for Wildfires. The Seventeenth International Workshop on Constraint Modelling and Reformulation (ModRef 2018), Aug 2018, Lille, France. 17p.

[2] H.W. Hamacher and S.A. Tjandra. Mathematical modelling of evacuation problems - a state of the art. Pedestrian and Evacuation Dynamics, pages 227-266, 2002. Springer Verlag.

[3] Madkour, Amgad, Walid G. Aref, Faizan Ur Rehman, Mohamed Abdur Rahman and Saleh M. Basalamah. A Survey of Shortest-Path Algorithms. CoRR, abs/1705.02044, 2017

[4] Zwick, Uri. "Exact and approximate distances in graphs - a survey." In European Symposium on Algorithms, pp. 33-48. Springer, Berlin, Heidelberg, 2001.

[5] Sommer, Christian. "Shortest-path queries in static networks." ACM Computing Surveys (CSUR) 46, no. 4 (2014): 45.

[6] Chang Liu, Zhan-li Mao, Zhi-min Fu, Emergency Evacuation Model and Algorithm in the Building with Several Exits, Procedia Engineering, Volume 135, 2016, Pages 12-18, ISSN 1877-7058.

[7] $\mathrm{Pu}$, Shi, and Sisi Zlatanova. "Evacuation route calculation of inner buildings." In Geo-information for disaster management, pp. 11431161. Springer, Berlin, Heidelberg, 2005.

[8] Pursals, Salvador Casadesús, and Federico Garriga Garzón. "Optimal building evacuation time considering evacuation routes." European Journal of Operational Research 192, no. 2 (2009): 692-699.

[9] Barnes, Matthew and Leather, Hugh and Arvind, D. (2007). Emergency Evacuation using Wireless Sensor Networks. Proceedings - Conference on Local Computer Networks, LCN. 851-857. 10.1109/LCN.2007.48.

[10] Shekhar, Shashi, KwangSoo Yang, Venkata MV Gunturi, Lydia Manikonda, Dev Oliver, Xun Zhou, Betsy George, Sangho Kim, Jeffrey MR Wolff, and Qingsong Lu. "Experiences with evacuation route planning algorithms." International Journal of Geographical Information Science 26, no. 12 (2012): 2253-2265.

[11] Zhou, Xun, Betsy George, Sangho Kim, Jeffrey MR Wolff, Qingsong Lu, Shashi Shekhar, and O. Nashua. "Evacuation Planning: A Spatial Network Database Approach.” IEEE Data Eng. Bull. 33, no. 2 (2010): 26-31.

[12] K. Yang et al., "Intelligent Shelter Allotment for Emergency Evacuation Planning: A Case Study of Makkah." HajjCore tech. report P1104-T1, 2012; http://docs.lib.purdue.edu/cctech/9

[13] Yang, KwangSoo, Apurv Hirsh Shekhar, Faizan Ur Rehman, Hatim Lahza, Saleh Basalamah, Shashi Shekhar, Imtiaz Ahmed, and Arif Ghafoor. "Intelligent shelter allotment for emergency evacuation planning: A case study of makkah." IEEE Intelligent Systems 30, no. 5 (2015): 66-76.

[14] Louai Alarabi, Ahmed Eldawy, Rami Alghamdi, and Mohamed F. Mokbel. 2014. TAREEG: a MapReduce-based system for extracting spatial data from OpenStreetMap. In Proceedings of the 22nd ACM SIGSPATIAL International Conference on Advances in Geographic Information Systems (SIGSPATIAL '14). ACM, New York, NY, USA, 83-92. DOI: http://dx.doi.org/10.1145/2666310.2666403

[15] D. Helbing, A. Johansson and H. Z. Al-Abideen, The Dynamics of Crowd Disasters: An Empirical Study. Physical Review E 75: 046109, 2007. (www.trafficforum.ethz.ch/crowdturbulence/)

[16] R.L. Hughes, "The Flow of Human Crowds", Annual Review of Fluid Mechanics, 35, 169-182 (2003) 


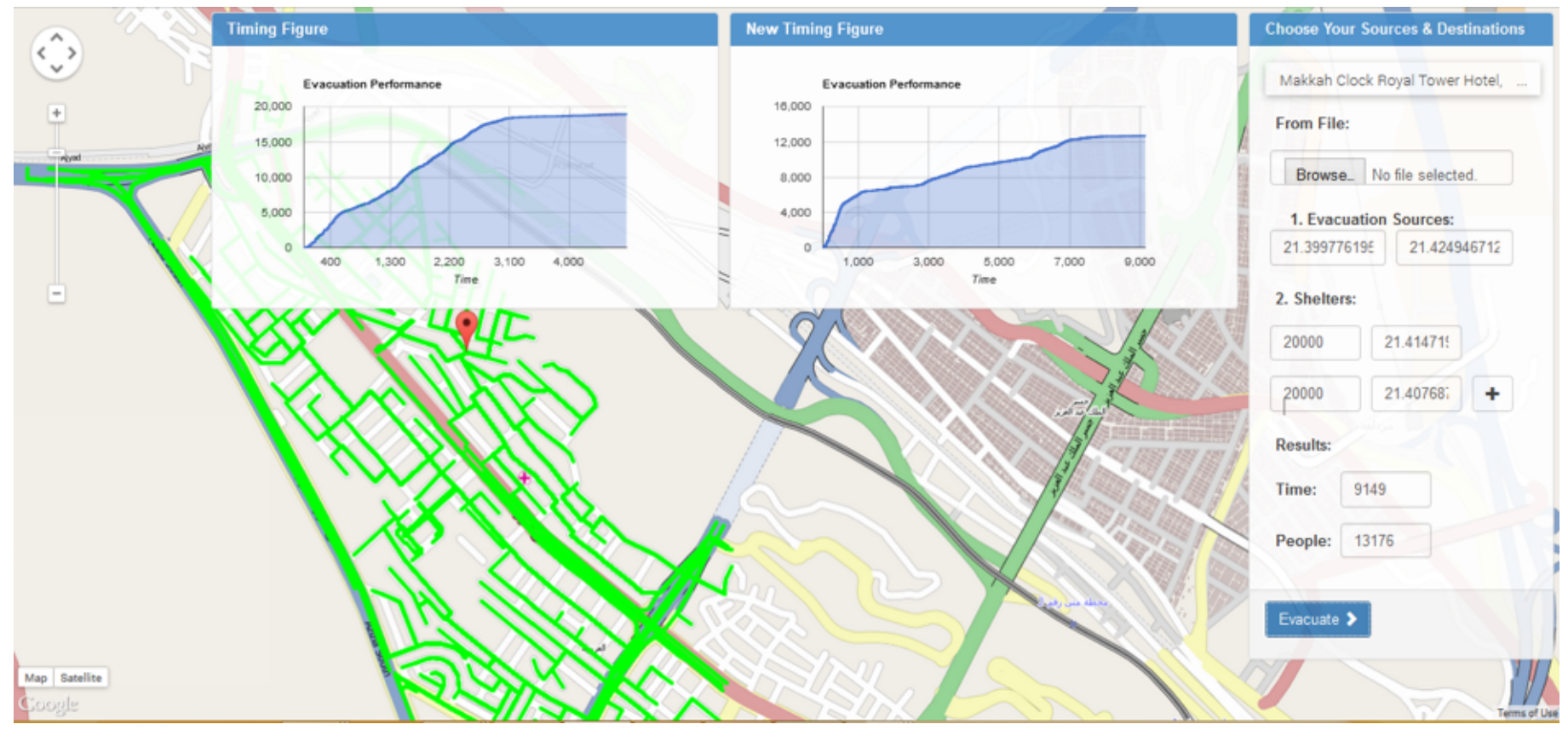

Fig. 7. Effect of blocking/expanding roads on evacuation time. Roads with red color are marked as closed.

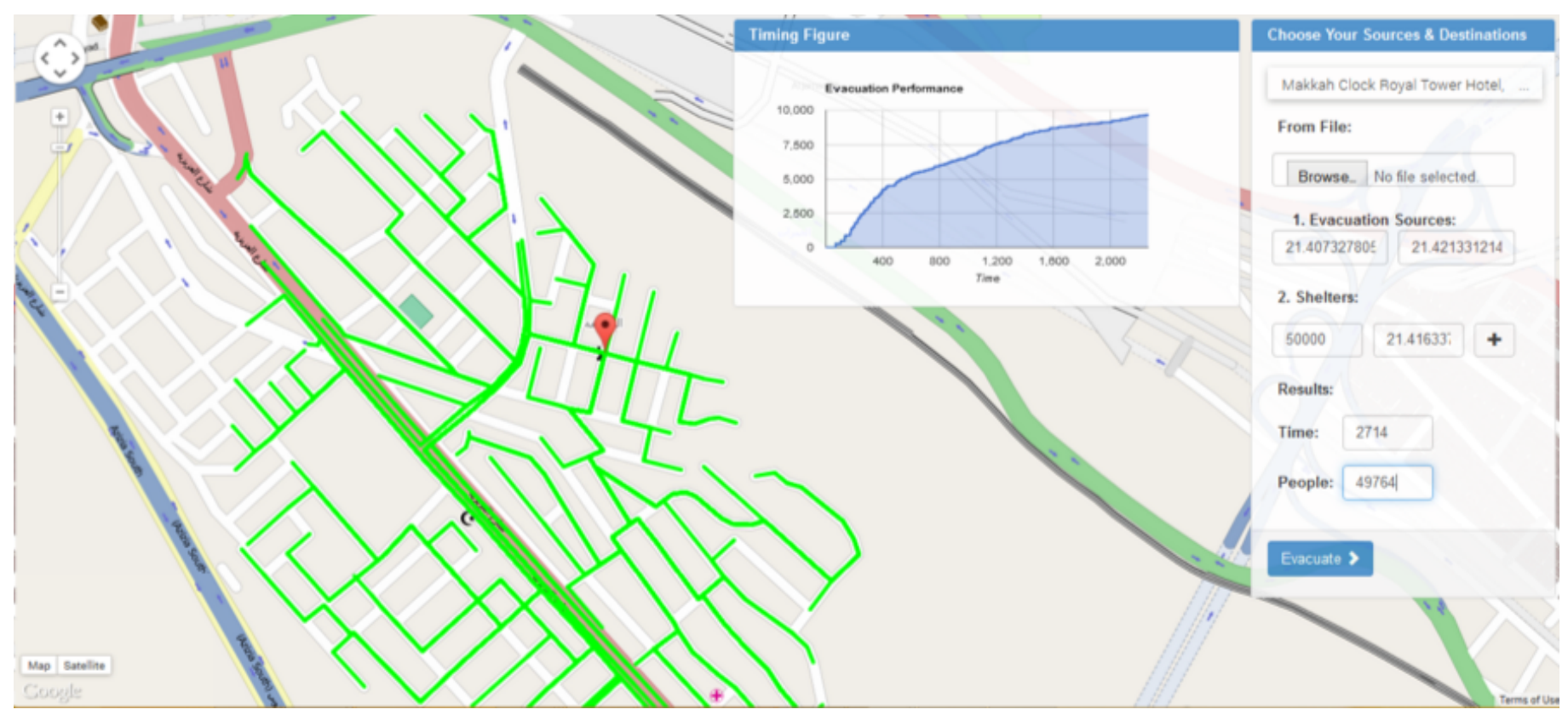

Fig. 8. Effect of increasing the number of evacuees. 


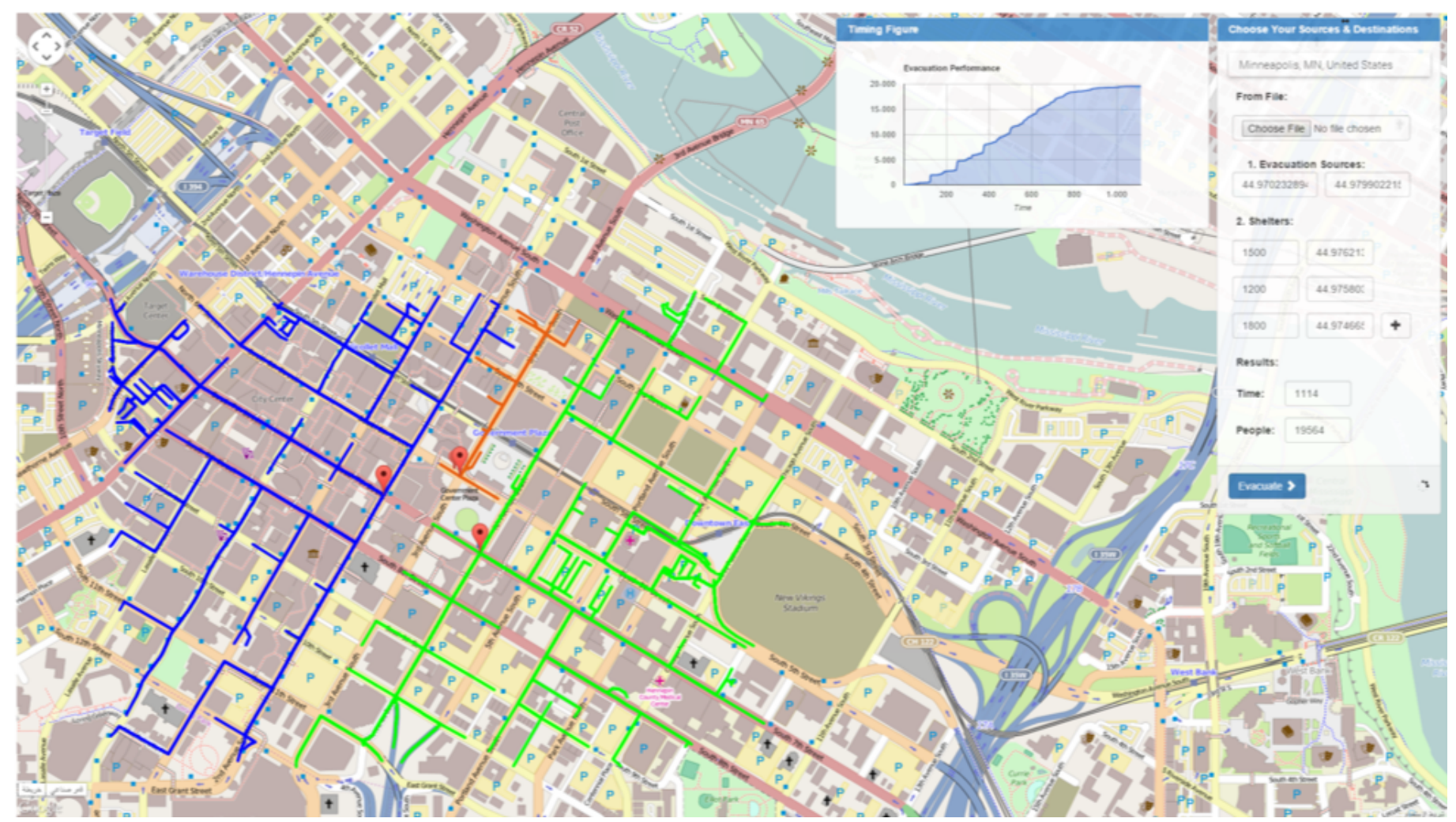

Fig. 9. Effect of clustering shelters.

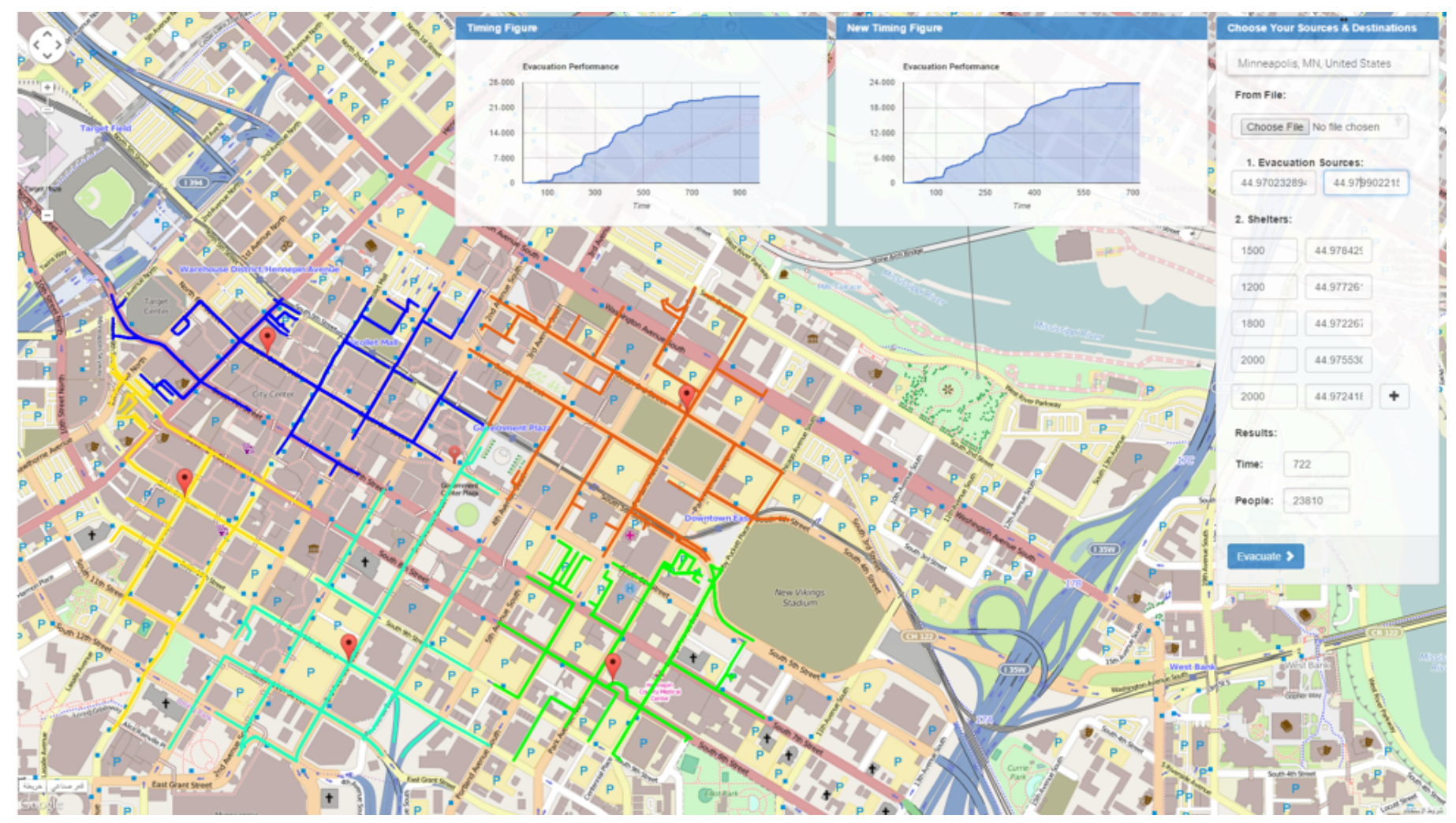

Fig. 10. Effect of spreading shelters. 


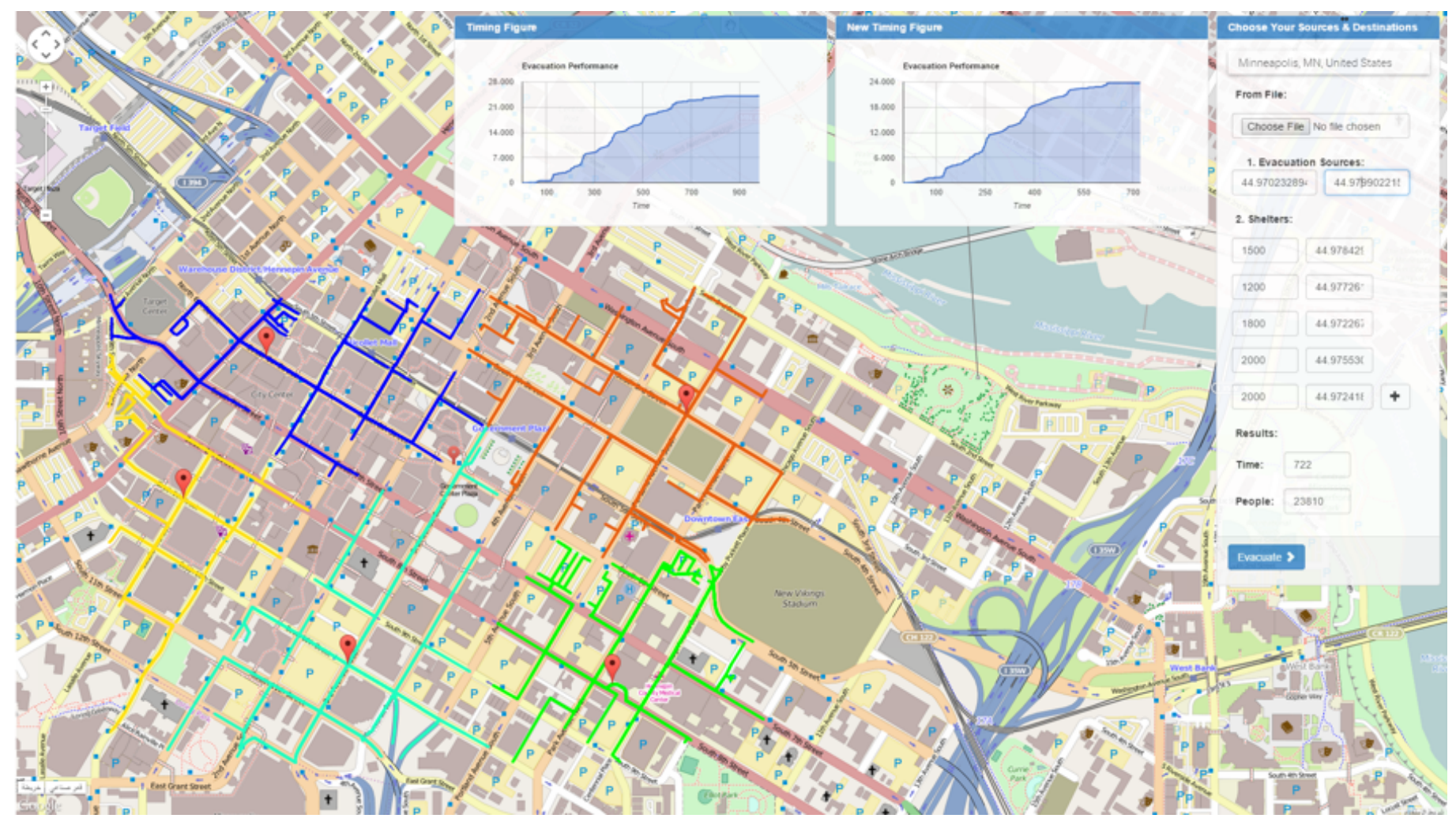

Fig. 11. Effect of increasing the number of shelters. 